\title{
Lightweight Document Clustering
}

\author{
Sholom M. Weiss, Brian F. White, and Chidanand V. Apte \\ IBM T.J. Watson Research Center \\ P.O. Box 218, Yorktown Heights, NY 10598, USA \\ sholom@us.ibm.com, bfwhite@us.ibm.com, apte@us.ibm.com
}

\begin{abstract}
A lightweight document clustering method is described that operates in high dimensions, processes tens of thousands of documents and groups them into several thousand clusters, or by varying a single parameter, into a few dozen clusters. The method uses a reduced indexing view of the original documents, where only the $\mathrm{k}$ best keywords of each document are indexed. An efficient procedure for clustering is specified in two parts (a) compute $\mathrm{k}$ most similar documents for each document in the collection and (b) group the documents into clusters using these similarity scores. The method has been evaluated on a database of over 50,000 customer service problem reports that are reduced to 3,000 clusters and 5,000 exemplar documents. Results demonstrate efficient clustering performance with excellent group similarity measures.
\end{abstract}

\section{Introduction}

The objective of document clustering is to group similar documents together, assigning them to the same implicit topic. Why is document clustering of interest? The original motivation was to improve the effectiveness of information retrieval. Standard information retrieval techniques, such as nearest neighbor methods using cosine distance, can be very efficient when combined with an inverted list of word to document mappings. These same techniques for information retrieval perform a variant of dynamic clustering, matching a query or a full document to their most similar neighbors in the document database. Thus standard information retrieval techniques are efficient and dynamically find similarity among documents, reducing the value for information retrieval purposes of finding static clusters of large numbers of similar documents [Sparck-Jones, 1997].

The advent of the web has renewed interest in clustering documents in the context of information retrieval. Instead of pre-clustering all documents in a database, the results of a query search can be clustered, with documents appearing in multiple clusters. Instead of presenting a user with a linear list of related documents, the documents can be grouped in a small number of clusters, perhaps ten, and the user has an overview of different documents that have been found in the search and their relationship within similar groups of documents. One approach to this type of visualization and presentation is described in [Zamir et al., 1997]. Here again though, the direct retrieval and linear list remains effective, especially when the user is given a "more like this" option that finds a subgroup of documents representing the cluster of interest to the user.

Document clustering can be of great value for tasks other than immediate information retrieval. Among these task are 
- summarization and label assignment, or

- dimension reduction and duplication elimination.

Let's look at these concepts by way of a help-desk example, where users submit problems or queries online to the vendor of a product. Each submission can be considered a document. By clustering the documents, the vendor can obtain a overview of the types of problems the customers are having, for example a computer vendor might discover that printer problems comprise a large percentage of customer complaints. If the clusters form natural problem types, they may be assigned labels or topics. New user problems may then be assigned a label and sent to the problem queue for appropriate response. Any number of methods can be used for document categorization once the appropriate clusters have been identified. Typically, the number of clusters or categories number no more than a few hundred and often less than 100.

Not all users of a product report unique problems to the help-desk. It can be expected that most problem reports are repeat problems, with many users experiencing the same difficulty. Given enough users who report the same problem, an FAQ, Frequently Asked Questions report, may be created. To reduce the number of documents in the database of problem reports, redundancies in the documents must be detected. Unlike the summary of problem types, many problems will be similar but still have distinctions that are critical. Thus, while the number of clusters needed to eliminate duplication of problem reports can be expected to be much smaller than the total number of problems reports, the number of clusters is necessarily relatively large, much larger than needed for summarization of problem types.

In this paper, we describe a new lightweight procedure for clustering documents. It is intended to operate in high dimensions with tens of thousands of documents and is capable of clustering a database into the moderate number of clusters need for summarization and label assignment or the very large number of clusters needed for the elimination of duplication.

\section{Document Clustering Techniques}

The classical k-means technique [Hartigan and Wong, 1979] can be applied to document clustering. Its weaknesses are well known. The number of clusters $\mathrm{k}$ must be specified prior to application. The summary statistic is a mean of the values for each cluster. The individual members of the the cluster can have a high variance and the mean may not be a good summary of the cluster members. As the number of clusters grow, for example to thousands of clusters, k-means clustering becomes untenable, approaching the $O\left(n^{2}\right)$ comparisons where $\mathrm{n}$ is the number of documents. However, for relatively few clusters and a reduced set of pre-selected words, k-means can do well[Vaithyanathan and Dom, 1999].

More recent attention has been given to hierarchical agglomerative methods [Griffiths et al., 1997]. The documents are recursively merged bottom up, yielding a decision tree of recursively partitioned clusters. The distance measures used 
to find similarity vary from single-link to more computationally expensive ones, but they are closely tied to nearest-neighbor distance. The algorithm works by recursively merging the single best pair of documents or clusters, making the computational costs prohibitive for document collections numbering in the tens of thousands.

To cluster very large numbers of documents, possibly with a large number of clusters, some compromises must be made to reduce the number of indexed words and the number of expected comparisons. In [Larsen and Aone, 1999], indexing of each document is reduced to the 25 highest scoring TF-IDF words (term frequency and inverse document frequency [Salton and Buckley, 1997], and then $\mathrm{k}$-means is applied recursively, for $\mathrm{k}=9$. While efficient, this approach has the classical weaknesses associated with k-means document clustering. A hierarchical technique that also works in steps with a small, fixed number of clusters is described in [Cutting et al., 1992].

We will describe a new lightweight procedure that operates efficiently in high dimensions and is effective in directly producing clusters that have objective similarity. Unlike k-means clustering, the number of clusters is dynamically determined, and similarity is based on nearest-neighbor distance, not mean feature distance. Thus, the new document clustering method maintains the key advantage of hierarchical clustering techniques, their compatibility with information retrieval methods, yet performance does not rapidly degrade for large numbers of documents.

\section{Methods and Procedures}

\subsection{Data Preparation}

Clustering algorithms process documents in a transformed state, where the documents are represented as a collection of terms or words. A vector representation is used: in the simplest format, each element of the vector is the presence or absence of a word. The same vector format is used for each document; the vector is a space taken over the complete set of words in all documents. Clearly, a single document has a sparse vector over the set of all words. Some processing may take place to stem words to their essential root and to transform the presence or absence of a word to a score, such as TF-IDF, that is a predictive distance measure. In addition weakly predictive words, stopwords, are removed. These same processes can be used to reduce indexing further by measuring for a document's vector only the top k-words in a document and setting all remaining vector entries to zero.

An alternative approach to selecting a subset of features for a document, described in [Weiss et al., 2000], assumes that documents are carefully composed and have effective titles. Title words are always indexed along with the k most frequent words in the document and any human-assigned key words.

Not all words are of the same predictive value and many approaches have been tried to select a subset of words that are most predictive. The main concept 
is to reduce the number of overall words that are considered, which reduces the representational and computational tasks of the clustering algorithm. Reduced indexing can be effective in these goals when performed prior to clustering. The clustering algorithm accepts as input the transformed data, much like any information retrieval system, and works with a vector representation that is a transformation of the original documents.

\subsection{Clustering Methods}

Our method uses a reduced indexing view of the original documents, where only the $\mathrm{k}$ best keywords of each document are indexed. That reduces a document's vector size and the computation time for distance measures for a clustering method. Our procedure for clustering is specified in two parts (a) compute $\mathrm{k}$ most similar documents (typically the top 10) for each document in the collection and (b) group the documents into clusters using these similarly scores. To be fully efficient, both procedures must be computationally efficient. Finding and scoring the $\mathrm{k}$ most similar documents for each document will be specified as a mathematical algorithm that processes fixed scalar vectors. The procedure is simple, a repetitive series of loops that accesses a fixed portion of memory, leading to efficient computation. The second procedure uses the scores for the $\mathrm{k}$ most similar documents in clustering the document. Unlike the other algorithms described earlier, the second clustering step does not perform a "best-match firstout" merging. It merges documents and clusters based on a "first-in first-out" basis.

Table 3.2 describes the data structures needed to process the algorithms. Each of these lists can be represented as a simple linear vector. Table 3.2 describes the steps for the computation of the $\mathrm{k}$ most similar documents, typically the top 10, for each document in the collection. Similarity or distance is measured by a simple additive count of words found in both documents that are compared plus their inverse document frequency. This differs from the standard TF-IDF formula in that term frequency is measured in binary terms, i.e. 0 or 1 for presence or absence. In addition the values are not normalized, just the sum is used. In a comparative study, we show that TF-IDF has slightly stronger predictive value, but the simpler function has numerous advantages in terms of interpretability, simple additive computation, and elimination of storage of term frequencies. The steps in Table 3.2 can readily be modified to use TF-IDF scoring.

The remaining task is to group the documents into clusters using these similarly scores. We describe a a single pass algorithm for clustering, with at most $\mathrm{k}^{*} \mathrm{n}$ comparisons of similarity, where $\mathrm{n}$ is the number of documents.

For each document $D_{i}$, the scoring algorithm produces a set of k documents, $\left\{D_{j}\right\}$, where $\mathrm{j}$ varies from 1 to $\mathrm{k}$. Given the scores of the top-k matches of each document $D_{i}$, Table 3.2 describes the actions that may be taken for each matched pair during cluster formation. Documents are examined in a pairwise fashion proceeding with the first document and its top-k matches. Matches below a preset minimum score threshold are ignored. Clusters are formed by the document 
doclist: The words (terms) in each document. A series of numbers; documents are separated by zeros. example: Sequence $=\begin{array}{lllll}10 & 44 & 98 & 0 & 24\end{array}$. The first document has words 10, 44 and 98. The second document has words 24 ..

wordlist: The documents in which a word is found. A series of consecutive numbers pointing to specific document numbers.

word(c): A pointer to wordlist indicates the starting location of the documents for word c. To process all documents for word c, access word(c) through word $(c+1)-1$. example: $\operatorname{word}(1)=1, \operatorname{word}(2)=4 ;$ wordlist $=\left\{\begin{array}{lllll}18 & 22 & 64 & 16 & \ldots\end{array}\right\}$ Word 1 appears in the documents listed in locations 1,2 , and 3 in wordlist. The documents are 18, 22, and 64 .

$\mathbf{p v}(\mathbf{c})$ : predictive values of word $\mathrm{c}=1+\mathrm{idf}$, where idf is $1 /$ (number of documents where word c appears)

Table 1. Definitions for Top- $k$ Scoring Algorithm

1. Get the next document's words (from doclist), and set all document scores to zero.

2. Get the next word, w, for current document. If no words remain, store the $\mathrm{k}$ documents with the highest scores and continue with step (i).

3. For all documents having word w (from wordlist), add to their scores and continue with step (ii).

Table 2. Steps for Top-k Scoring Algorithm

pairs not yet in clusters. Clusters are merged when the matched pair appear in separate clusters. As we shall see in Section 4, not allowing merging yields a very large number clusters whose members are highly similar. The single setting of the minimum score has a strong effect on the number of clusters; a high value produces a relatively large number of clusters and a zero value produces a relatively small number of clusters. Similarly, a high minimum score may leave some documents unclustered, while a low value clusters all documents. As an alternative to merging, it may be preferable to repeat the same document in multiple clusters. We do not report results on this form of duplication, typically done for smaller numbers of documents, but the procedure provides an option for duplicating documents across clusters.

\subsection{Measures for Evaluation of Clustering Results}

How can we objectively evaluate clustering performance? Very often, the objective measure is related to the clustering technique. For example, k-means clustering can measure overall distance from the mean. Techniques that are based on nearest neighbor distance, such as most information retrieval techniques, can measure distance from the nearest neighbor or the average distance from other cluster members.

For our clustering algorithm, distance is measured mostly in terms of counts of words present in documents. A natural measure of cluster performance is 
670 S.M. Weiss, B.F. White, and C.V. Apte

1. If score for $D_{i}$ and $D_{j}$ is less than Minimum Score, next pair.

2. If $D_{i}$ and $D_{j}$ are already in the same cluster, next pair.

3. If $D_{i}$ is in a cluster and $D_{j}$ isn't, add $D_{j}$ to the $D_{i}$ cluster, next pair.

4. Cluster Merge Step: if both $D_{i}$ and $D_{j}$ are in separate clusters:

(a) If action plan is "no merging", next pair.

(b) If action plan is "repeat documents", repeat $D_{j}$ in all the $D_{i}$ clusters, next pair.

(c) Merge the $D_{i}$ cluster with $D_{j}$ cluster, next pair.

Table 3. Actions for Clustering Document Pairs

the average number of indexed words per cluster, i.e. the local dictionary size. Analogous measures of cluster "cohesion," that count the number common words among documents in a cluster, have been used to evaluate performance[Zamir et al., 1997]. The average is computed by weighing the number of documents in the cluster as in equation 1 , where $\mathrm{N}$ is the total number number of documents, $\mathrm{m}$ is the number of clusters, $S_{i z e_{k}}$ is the number of documents in the k-th cluster, and $L$ Dict $_{k}$ is the number of indexed words in the $\mathrm{k}$-th cluster.

$$
\text { AverageDictionarySize }=\sum_{k=1}^{m} \frac{\text { Size }_{k}}{N} \cdot \text { LDict }_{k}
$$

Results of clustering are compared to documents randomly assigned to the same size clusters. Clearly, the average dictionary size for computed clusters should be much smaller than those for randomly assigned clusters of the same number of documents.

\subsection{Summarizing Clustering Results}

The same measure of evaluation can be used to find exemplar documents for a cluster. The local dictionary of a document cluster can be used as a virtual document that is matched to the members of the cluster. The top-k matched documents can be considered a ranked list of exemplar documents for the cluster.

Selecting exemplar documents from a cluster is a form of summary of the cluster. The technique for selecting the exemplars is based on matching the cluster's dictionary of words to its constituent documents. The words themselves can provide another mode of summary for a cluster. The highest frequency words in the local dictionary of a cluster often can distinguish a cluster from others. If only a few words are extracted, they may be considered a label for the cluster.

\section{Results}

To evaluate the performance of the clustering algorithms, we obtained 51,110 documents, taken from reports from customers having IBM AS/400 computer 
systems. These documents were constructed in real-time by customer service representatives who record their phone dialog with customers encountering problems with their systems.

The documents were indexed with a total of 21,682 words in a global dictionary computed from all the documents. Table 4 summarizes the results for clustering the document collection in terms of the number of clusters, the average cluster size, the ratio of the local dictionary size to random assignment, the percentage of unclustered documents, the minimum score for matching document pairs, and whether merging was used. The first row in the table indicates that 49 clusters were found with an average size of 1027 documents. A random cluster's dictionary was on average 1.4 times larger than the generated cluster; and $1.5 \%$ of the documents were not clustered. These results were obtained by using a minimum score of 1 and cluster merging was allowed. All results are for finding the top-10 document matches.

\begin{tabular}{|c|c|c|c|c|c|}
\hline Cnum & AveSize & RndRatio & Unclust \% & MinScore & Merge \\
\hline 49 & 1027.3 & 1.4 & 1.5 & 1 & yes \\
\hline 86 & 579.6 & 1.4 & 2.5 & 2 & yes \\
\hline 410 & 105.5 & 1.5 & 16.2 & 3 & yes \\
\hline 3250 & 15.5 & 1.8 & 1.5 & 1 & no \\
\hline 3346 & 14.9 & 1.8 & 2.5 & 2 & no \\
\hline 3789 & 11.4 & 1.9 & 16.2 & 3 & no \\
\hline
\end{tabular}

Table 4. Results for Clustering Help-Desk Problems

A single clustering run, one row in Table 4 currently takes 15 minutes on a $375 \mathrm{MHz}$ RS6000 running AIX. The code is written in Java.

Exemplar documents were selected for each of the 3250 clusters found in the fourth entry of the table. For some large clusters, two or three exemplars were selected for a total of 5,000 exemplar documents. Using the same scoring scheme, each of the exemplars was matched to the original 51,110 documents. $98.4 \%$ of the documents matched at least one of of the exemplars, having at least one indexed word in common. $60.7 \%$ of the documents matched an exemplar of their assigned cluster, rather than an exemplar of an alternative cluster.

\section{Discussion}

The lightweight document clustering algorithms achieves our stated objectives. The process is efficient in high dimensions, both for large document collections and for large numbers of clusters. No compromises are made to partition the clustering process into smaller sub-problems. All documents are clustered in one stage.

These clustering algorithms have many desirable properties. Unlike k-means clustering, the number of clusters is dynamically assigned. A single parameter, 
the minimum score threshold, effectively controls whether a large number of clusters or a much smaller number is chosen. By disallowing merging of clusters, we are able to obtain a very large number of clusters.

The success of clustering can be measured in terms of an objective function. In our case, we are using the local dictionary size of the cluster. In all instances, we see that clustering is far better than random assignment. As expected, the greater the number of clusters, the better the performance when measured by dictionary size. In the help-desk application, it is important to remove duplication, while still maintaining a large number of exemplar documents. The help-desk clusters have strong similarity for their documents, suggesting that they can be readily summarized by a one or two documents. For the largest number of clusters, dictionary size is nearly half that for random document assignment, far better than for smaller number of clusters.

The help-desk application is characterized by a large number of specialized indexed words for computer systems. Future applications will determine the generality of this approach. There is ample room for enhancements to the computer implementation that will lead to faster performance and a capability to run on far larger document collections.

\section{References}

[Cutting et al., 1992] D. Cutting, D. Karger, J. Pedersen, and J. Tukey. Scatter/Gather: a Cluster-based Approach to Browsing Large Document collections. In Proceedings of the 15th ACM SIGIR. ACM, 1992.

[Griffiths et al., 1997] A. Griffiths, H. Luckhurst, and P. Willett. Using interdocument similarity information in document retrieval systems. In P. Sparck-Jones, K. and. Willet, editor, Readings in Information Retrieval, pages 365-373. Morgan Kaufmann, 1997.

[Hartigan and Wong, 1979] J. Hartigan and M Wong. A k-means clustering algorithm. Applied Statitsics, 1979.

[Larsen and Aone, 1999] B. Larsen and C. Aone. Fast and Effective Text Mining Using Linear-time Document Clustering. In Proceedings of the 5th International Conference on Knowledge Discovery ad Data Mining, pages 16-22. ACM, 1999.

[Salton and Buckley, 1997] G. Salton and C. Buckley. Term-weighting approaches in automatic text retrieval. In P. Sparck-Jones, K. and. Willet, editor, Readings in Information Retrieval, pages 323-328. Morgan Kaufmann, 1997.

[Sparck-Jones, 1997] P. Sparck-Jones, K. and. Willet. Chapter 6 - techniques. In P. Sparck-Jones, K. and. Willet, editor, Readings in Information Retrieval, pages 305-312. Morgan Kaufmann, 1997.

[Vaithyanathan and Dom, 1999] S. Vaithyanathan and B. Dom. Model Selection in Unsupervised Learning with Applications to Document Clustering. In Proceedings International Conference on Machine Learning, 1999.

[Weiss et al., 2000] S. Weiss, B. White, C. Apté, and F. Damerau. Lightweight document matching for help-desk applications. IEEE Intelligent Systems, page in press, 2000 .

[Zamir et al., 1997] O. Zamir, O. Etzioni, O. Madani, and R. Karp. Fast and Intuitive Clustering of Web Documents. In Proceedings of the 3rd International Conference on Knowledge Discovery ad Data Mining. Morgan Kaufmann, 1997. 